\title{
ON COMPACT ACTION IN JB-ALGEBRAS
}

\author{
by L. J. BUNCE
}

(Received 16th August 1982)

\section{Introduction}

A real Jordan algebra which is also a Banach space with a norm which satisfies

$$
\left\|a^{2}-b^{2}\right\| \leqq \max \left(\left\|a^{2}\right\|,\left\|b^{2}\right\|\right), \quad\left\|a^{2}\right\|=\|a\|^{2}
$$

for each pair $a, b$ of elements, is said to be a $J B$-algebra. A $J B$-algebra which is also a Banach dual space is said to be a $J B W$-algebra.

Important examples of $J B$-algebras include the $J C$-algebras, these are by definition the uniformly closed Jordan subalgebras of the Jordan algebra of all bounded selfadjoint operators on a complex Hilbert space, and also the algebra $M_{3}^{8}$ of hermitian $3 \times 3$ matrices over the octonions. A $J C$-algebra which is closed in the weak operator topology is a $J B W$-algebra said to be a $J W$-algebra.

The reader is referred to $[2-3,7-9,11,14-15,18]$ for the relevant background on $J B$ algebras and to $[10,16-17]$ for that on $J C$ algebras. A detailed account of the general theory of Jordan algebras is to be found in [12].

In the $J B$-algebra $A$, the Jordan triple product $\{a, b, c\}$, of elements $a, b$ and $c$ in $A$, is defined by $\{a, b, c\}=(a \circ b) \circ c+a \circ(b \circ c)-(a \circ c) \circ b$, and for each element $a$ in $A$, the operators on $A, U_{a}$ and $L_{a}$ are defined by $U_{a}(b)=\{a, b, a\}, L_{a}(b)=a \circ b$ for each element $b$ in $A$. When $A$ is a $J C$-algebra these operations reduce to

$$
L_{a}(b)=\frac{1}{2}(a b+b a), \quad U_{a}(b)=a b a, \quad\{a, b, c\}=\frac{1}{2}(a b c+c b a)
$$

For the $J B$-algebra $A, A^{+}$, the set of squares of elements of $A$, is a positive cone which generates $A$. A $J B$-subalgebra $B$ of $A$ is said to be an hereditary $J B$-subalgebra of $A$ if whenever $0 \leqq a \leqq b$ with $a \in A$ and $b \in B$, then $a \in A$. Also, a linear subspace, $J$, of $A$, is said to be a Jordan (resp: quadratic) ideal of $A$ if $L_{x}(A)$ (resp: $U_{x}(A)$ ) is contained in $A$ for every element $x$ of $J$. A fact that will frequently be used is that the uniformly closed quadratic ideals of $A$ are precisely the hereditary $J B$-subalgebras of $A$ [8, Theorem 2.3]. The $J B$-algebra $A$ will habitually be considered to be a $J B$-subalgebra of the $J B W$ algebra $A^{* *}$, the second dual of $A$ (see $[11,14-15]$ ), and by $\tilde{A}$ will be meant the $J B$ subalgebra of $A^{* *}$ generated by $A$ and the unit, 1 , of $A^{* *}$.

The purpose of this note is to identify, in an arbitrary $J B$-algebra $A$, those sets of elements

$$
x_{1} \text { (resp: } x_{2} \text { ) of } A \text { for which } U_{x_{1}} \text { (resp: } L_{x_{2}} \text { ) are weakly compact on } A \text {. }
$$


and,

$$
\left.x_{3} \text { (resp: } x_{4}\right) \text { of } A \text { for which } U_{x_{3}}\left(\text { resp: } L_{x_{4}} \text { ) are compact on } A\right. \text {. }
$$

By way of justification, we might mention the fact that though in a $C^{*}$ algebra $A$ it is easily seen, by elementary analysis, that the set of elements $x$ of $A$ for which the operator on $A, a \mapsto a x a$, is compact is a two-sided ideal of $A$ and, in view of the results of [1], consequently recognisable as the largest dual (in the sense of $[5,4.7 .20]$ ) twosided ideal in $A$, the corresponding method of insight is not available for $J B$ algebras. Indeed, the former of the sets of (II), above, is not, at times, even a linear space. A similar difficulty arises with the latter set of (II).

\section{On associative subalgebras in a $J B$ algebra}

Recall that an associative $J B$-algebra can be realised as the self-adjoint part of a commutative $C^{*}$-algebra [1, Proposition 2.3, 14, Lemma 2.2], and that, in particular, for each element $a$ in the $J B$ algebra $A$ the (associative) $J B$-subalgebra, $C(a)$, of $A$ generated by $a$ can be identified with the algebra of all continuous real valued functions vanishing at infinity on the locally compact Hausdorff space $\sigma(a)$, of all real numbers $\lambda$ for which $a-\lambda 1$ is not invertible in $\tilde{A}$. Recall also that for each pair of elements $a, b$ in the $J B$ algebra $A, C(a, b)$, the $J B$ subalgebra of $A$ generated by $a$ and $b$ can be realised as a $J C$ algebra, by [18, Proposition 2.1]. It is said that the pair $a, b$ operator commute in $A$ if $L_{a} L_{b}=L_{b} L_{a}$ on $A$. The set of all elements of $A$ which operator commute with every element in $A$ is said to be the centre, $Z(A)$, of $A$.

In this preliminary section two lemmas are proved, the first of which is a slight variation of a result of Youngson [19, Theorem 5]. The result is known for $J C$-algebras [16, Proposition 1]. The following Jordan identities will be needed (see [12, page 37]) in Lemma 2 and after.

$$
\begin{gathered}
U_{U_{a}(b)}=U_{a} U_{b} U_{a} \\
\left(U_{a}(b)\right)^{2}=U_{a} U_{b}\left(a^{2}\right) .
\end{gathered}
$$

Lemma 1. Let $A$ be a JB algebra and let $a, b$ be elements of $A$. Then the following are equivalent:

(i) $U_{a}(b)=a^{2} \circ b$.

(ii) $a$ and $b$ operator commute in $A$.

(iii) $C(a, b)$ is associative.

Proof. From [16, Proposition 1] and [18, Proposition 2.1] it follows that (i) and (iii) are equivalent and that (ii) implies (i). It remains to prove that (i) implies (ii). In order to achieve this it is enough to suppose that $A$ equals $M_{3}^{8}$, as can be seen on application of [16, Proposition 1] together with the fact that $A$ has a faithful family of representations each member of which maps $A$ onto $M_{3}^{8}$ or a $J C$-algebra. But then $C(a, b)$ is finite dimensional and so, in particular, $a$ is a finite linear combination of 
projections of $C(a, b)$. The desired conclusion now follows on application of (i) $\Leftrightarrow$ (ii) and [2, Lemma 2.11].

A projection $p$ in the $J B$-algebra $A$ is said to be a one-dimensional projection of $A$ if $U_{p}(A)$ has dimension one.

Lemma 2. Let $A$ be a JB-algebra and let $B$ be a maximal associative JB-subalgebra of $A$. Then every one-dimensional projection of $B$ is also a one-dimensional projection of $A$.

Proof. In any associative algebra one has the identity

$$
(x y x) z+z(x y x)=(x z+z x) y x+x y(x z+z x)-x(y z+z y) x .
$$

By Macdonald's Theorem [12, page 41] the corresponding identity

$$
U_{x}(y) \circ z=2\{x \circ z, y, x\}-U_{x}(y \circ z)
$$

will hold in every Jordan algebra.

Let $a \in A, b \in B$ and let $e$ be a one-dimensional of $B$. Observe that $e \circ b=U_{e}(b)=\lambda e$, for some real number $\lambda$, and that $e$ and $b$ operator commute in $A$, by [2, Lemma 2.11] or Lemma 1, which in turn means that

$$
U_{e}(a \circ b)=U_{e}(a) \circ b=\lambda U_{e}(a)
$$

on applying (2.3). From (2.1), (2.2), it follows that

$$
\left(U_{e}(a)\right)^{2} \circ b=\left(U_{e} U_{a}(e)\right) \circ b=\lambda U_{e} U_{a}(e)=U_{U_{a}(e)}(b),
$$

where the second equality is obtained on replacing $a$ with $U_{a}(e)$ in (2.4). From Lemma 1 and the assumption on $B$ it follows that $U_{e}(A) \subset B$, as thus does the desired result.

\section{Compact action in $J B$ algebras}

A $J W$-algebra is said to be a $J W$ factor of Type $\mathrm{I}_{n}, n=\infty, 1,2,3, \ldots$, if it has trivial centre and contains a family, with cardinality $n$, of mutually orthogonal minimal projections with sum 1 . Recall $[2, \S 7]$ and [17], that the spin factors are precisely the $J W$ factors of Type $I_{2}$ and that for each spin factor $V$,

$$
V=\mathbb{R} 1 \oplus N(V), \quad N(V) \circ N(V)=\mathbb{R} 1,
$$

where $N(V)$ is the closed linear span of the non-trivial symmetries of $V$ (an element $s$ is said to be a non-trivial symmetry if $s \neq \pm 1, s^{2}=1$ ). Let us write, for each spin factor $V$,

$$
E(V)=\cup\left\{\mathbb{R} e ; e^{2}=e \neq 0,1\right\} .
$$

A $J B$-algebra $A$ is said to be $d u a l$, [4], if $\left(J^{0}\right)^{0}=J$ for every hereditary $J B$-subalgebra $J$ of $A$ (where given a subset $S$ of $A, S^{0}$ represents the annihilator of $S$ in $A$ ). The 
following discussion relies heavily upon the theory of dual $J B$ algebras and thus, for the convenience of the reader, some of the more immediately relevant properties are collected below in Theorem 3 .

The following terminology and notation is used below. The $J B-(\infty)$ sum of a family, $\left(A_{\lambda}\right)$, of $J B$ algebras, written $\left(\Sigma A_{\lambda}\right)_{0}$, is the $J B$-algebra of all generalised sequences $\left(x_{\lambda}\right)$, $\left(x_{\lambda} \in A_{\lambda}\right)$, vanishing at infinity, with norm $\left\|\left(x_{\lambda}\right)\right\|=\operatorname{Sup}\left\|x_{\lambda}\right\| ;$ in addition, if $F_{\lambda} \subset A_{\lambda}$, for each $\lambda$, defines a family of subsets, then $\left(\Sigma F_{\lambda}\right)_{0}$ will denote the set of the $\left(x_{\lambda}\right)$ in $\left(\Sigma A_{\lambda}\right)_{0}$ for which each $x_{\lambda}$ lies in $F_{\lambda}$. A $J B$-algebra is said to be simple if it contains no nontrivial norm closed Jordan ideals. Finally, given an arbitrary $J B$-algebra $A$, let $C(A)$ denote the closed linear span of the one-dimensional projections of $A$, and further, let

$$
\begin{aligned}
& C_{1}(A)=\left\{x \in A ; U_{x}: A \rightarrow A \text { is weakly compact }\right\}, \\
& C_{2}(A)=\left\{x \in A ; L_{x}: A \rightarrow A \text { is weakly compact }\right\}, \\
& C_{3}(A)=\left\{x \in A ; U_{x}: A \rightarrow A \text { is compact }\right\}, \\
& C_{4}(A)=\left\{x \in A ; L_{x}: A \rightarrow A \text { is compact }\right\} .
\end{aligned}
$$

Theorem 3. ([4, Sections 3-4])

(a) The simple dual JB-algebras are (up to isomorphism) precisely $M_{3}^{8}$, the spin factors, and the simple JC-algebras consisting of compact operators.

(b) The following are equivalent for the JB-algebra $A$ :

(i) A is a dual JB-algebra,

(ii) Each element of $A$ is of the form $\Sigma \lambda_{n} e_{n}$ (norm convergent), where $\left(e_{n}\right)$ is a sequence of mutually orthogonal one-dimensional projections of $A$,

(iii) $A$ is the $J B-(\infty)$ sum of a family of simple dual $J B$-algebras,

(iv) $A$ is an hereditary $J B$-subalgebra of $A^{* *}$,

(v) $U_{x}: A \rightarrow A$ is weakly compact for each $x$ in $A$,

(vi) $\quad L_{x}: A \rightarrow A$ is weakly compact for each $x$ in $A$.

Moreover, each of the above six conditions is equivalent to the condition (vii), below, if and only if $A$ has no representations onto an infinite dimensional spin factor:

(vii) $U_{x}: A \rightarrow A$ is compact for each $x$ in $A$.

A $J B$-algebra which satisfies condition (vii) of Theorem 3 is said to be a compact $J B$ algebra.

Lemma 4. Let $A$ be a $J B$-algebra. Then $C(A)$ is a Jordan ideal of $A$ and is the largest hereditary dual JB-subalgebra of $A$. It is also the largest hereditary JB-subalgebra of $A^{* *}$ contained in $A$.

Proof. Given an element $a$ of $A, U_{a}(C(A))$ is contained in $C(A)$, since for each onedimensional projection $e$ of $A$ there exists a one-dimensional projection $f$ of $A$ for which $U_{a}(e)=\left\|U_{a}(e)\right\| f$, by the argument of [4, Proposition 3.1], for example. It therefore 
follows, from [8, Lemma 2.4], that $C(A)$ is a Jordan ideal and, moreover, by [8, Theorem 2.3] and of Theorem 3 (b) (i) $\Leftrightarrow$ (ii), it is seen to be the largest hereditary dual $J B$-subalgebra of $A$. Finally, since any hereditary $J B$-subalgebra, $J$, of $A^{* *}$ which is contained in $A$ is, via the canonical embedding, an hereditary $J B$-subalgebra of $J^{* *}$, the last statement in the lemma follows from Theorem 3 (b) (i) $\Leftrightarrow$ (iv) and the first part of the proof.

Before stating the main theorem of this note, let us observe that for each element $x$ in the $J B$-algebra $A$, the norm closure, $A(x)$, of $U_{x}(A)$ is, by identity (2.1) and a simple limit argument, a quadratic ideal (and hence an hereditary $J B$-subalgebra) of $A$.

Writing $x=x_{+}-x_{-}$(where $x_{+}$and $x_{-}$lie in $C(x)^{+}, x_{+} \circ x_{-}=0$ ), it is easily seen, by spectral theory together with hereditary considerations, that both $x_{+}$and $x_{-}$, and hence $x$, lie (s) in $A(x)$.

Theorem 5. Let $A$ be a JB-algebra. Then

(i) $C_{1}(A), C_{2}(A)$ and the closed linear subspace generated by $C_{3}(A)$ are all equal to $C(A)$.

Moreover, on identifying $C(A)$ with the $J B-(\infty)$ sum of the family, $\left\{A_{\lambda} ; \lambda \in \Lambda\right\}$, of simple dual JB-algebras (justified by Theorem 3 and Lemma 4) and letting $F$ (resp: $G$ ) denote the set of those $\lambda \in \Lambda$ for which $A_{\lambda}$ is finite-dimensional (resp: an infinite-dimensional spin factor), one has, on retaining the above-mentioned identification,

(ii) $C_{3}(A)=\left(\sum_{\Lambda \backslash G} A_{\lambda}\right)_{0} \oplus\left(\sum_{G} E\left(A_{\lambda}\right)\right)_{0}$

(iii) $C_{4}(A)=\left(\sum_{F} A_{\lambda}\right)_{0} \oplus\left(\sum_{G} N\left(A_{\lambda}\right)\right)_{0}$.

Proof. (i) Let $x \in A$ and suppose that $U_{x}: A \rightarrow A$ is compact (resp: weakly compact). Then from identity (2.1), a simple limit argument, and well-known properties of compact (resp: weakly compact operators), [6, Chapter 6], for example, one has that $U_{y}: A(x) \rightarrow A(x)$ is compact (resp: weakly compact) for every element $y$ of $A(x)$. Therefore, $x \in A(x) \subset C(A)$, by Theorem 3 and Lemma 4 .

If $z$ lies in $A$ and $L_{z}: A \rightarrow A$ is weakly compact, let $B$ be a maximal associative subalgebra of $A$ containing $z$. Then $L_{z^{2}}=L_{z}^{2}=U_{z}$ on $B$ and so, by the above argument and the fact that $C(B)=C(A) \cap B$ (see Lemma 2), it follows that $z$ lies in $C(A)$. Thus $C_{3}(A) \subset C_{1}(A) \subset C(A)$ and $C_{2}(A) \subset C(A)$.

Conversely, suppose that $x$ lies in $C(A)$. By spectral theory, Lemma 4 and the equivalence of (i), (v) and (vi) of Theorem 3(b), $U_{x_{+}^{\frac{1}{2}}}, U_{x_{-}^{\frac{1}{+}}}, L_{x_{+}^{\frac{1}{2}}}, L_{x_{-}^{\frac{1}{ \pm}}}, L_{x_{+}}, L_{x_{-}}$are all weakly compact operators on $C(A)$ which, being a Jordan ideal of $A$, by Lemma 4 , implies that

$$
U_{x}=\left(U_{x_{+}^{\ddagger}}\right)^{2}+\left(U_{x_{-}^{\ddagger}}\right)^{2}-2 L_{x_{+}} L_{x_{-}}
$$


and consequently

$$
L_{x}=2\left(L_{x_{+}^{\frac{1}{4}}}\right)^{2}-U_{x_{+}^{\frac{1}{2}}}+2\left(L_{x_{-}^{\frac{1}{2}}}\right)^{2}-U_{x_{-}^{\frac{1}{2}}}
$$

are both weakly compact operators on $A$. Thus, $C_{3}(A) \subset C_{1}(A)=C_{2}(A)=C(A)$, and since all one-dimensional projections of $A$ must lie in $C_{3}(A)$, (i) results.

(ii) Certainly, $E\left(A_{\lambda}\right)$ is contained in $C\left(A_{3}\right)$ for each $\lambda$ in $G$ and from (i), together with Theorem 3(a), (b)(vii), one has that $A_{\lambda}=C_{3}\left(A_{\lambda}\right)$ for each $\lambda$ in $\Lambda \backslash G$. Thus given $\lambda$ in $\Lambda \backslash G$ and an element $x$ in $A_{\lambda}$, writing $|x|=x_{+}+x_{-}, x_{n}=(|x|+1 / n)^{-\frac{1}{2}}$, for each integer $n$, and operating in the associative $J B$-subalgebra $C(1, x)$ of $A$, one sees that $x_{+}^{2} \circ x_{n}$ converges uniformly to $x_{+}$(by Dini's Theorem, since $x_{+}^{2} \circ x_{n}$ increases to $x_{+}$). Similarly, $x_{-}^{2} \circ x_{n}$ converges uniformly to $x_{-}$. Hence,

$$
U_{x}=\lim U_{\left(x_{n} \circ|x|\right) \circ x}=\lim U_{x} U_{|x|} U_{x_{n}}
$$

acts compactly on the whole algebra $A$, since $A_{\lambda}$ is (identified with) a Jordan ideal of $A$. So, $A_{\lambda}=C_{3}\left(A_{\lambda}\right) \subset C_{3}(A)$ for each $\lambda$ in $\Lambda \backslash G$.

On the other hand, given an element $x$ of $C_{3}(A)$ and representing $x$ as an element $\left(x_{\lambda}\right)$ of the $J B-(\infty)$ sum of the family $\left\{A_{\lambda} ; \lambda \in \Lambda\right\}$, using (i), it is immediate that each $x_{\lambda}$ lies in $C_{3}\left(A_{\lambda}\right)$. Finally, since in a spin factor the norm and weak operator topologies coincide, [17, page 1060], and because $A\left(x_{\lambda}\right)$ is a compact hereditary $J B$-subalgebra of $A_{\lambda}$, by the first part of the proof of (i), it follows from Theorem 3(a), (b)(vii) together with [7, Theorem 2.3] that $x_{\lambda}$ lies in $E\left(A_{\lambda}\right)$ for each $\lambda$ in $G$. This completes the proof of (ii).

(iii) If $\lambda$ belongs to $G$, so that $V^{\circ}=A_{\lambda}$ is an infinite dimensional spin factor, then $N(V)$ $=C_{4}(V) \subset C_{4}(A)$. Indeed, the equality follows because $L_{s}(V)=\mathbb{R} 1+\mathbb{R} s$ for each symmetry $s \neq \pm 1$ in $V$, and the inclusion follows because (as one may check) $L_{s}^{3}=L_{s}$ for each such $s$.

If, on the other hand, $\lambda$ lies in $F$, then it is clear that $A=C_{4}\left(A_{\lambda}\right) \subset C_{4}(A)$, where the inclusion follows by an argument similar to that used in the last part of (i).

It is easy to see that if $\left(x_{\lambda}\right)$ is an element of $C(A)$ lying in $C_{4}(A)$ then each $x_{\lambda}$ lies in $C_{4}\left(A_{\lambda}\right)$. Thus the proof will be complete if, in these circumstances, it can be shown that whenever $x_{\lambda}$ is non-zero and $\lambda$ is not in $G$, then $\lambda$ is in $F$. In order to prove this, in view of Theorem 3(a), suppose that $B$ is a simple compact $J C$-algebra, contained in $B(H)$ for some complex Hilbert space $H$, for which there exists a non-zero element $x$ in $C_{4}(B)$. Let us define $T_{y}: B \rightarrow B(H)(b \mapsto y b)$, for each $y$ in $B$. Then, $T_{x}=2 T_{x} L_{x}-U_{x}: B \rightarrow B(H)$ is compact. It is seen therefore, from Theorem $3(\mathrm{~b})(\mathrm{i}) \Leftrightarrow(\mathrm{ii})$, that there exists a onedimensional projection $e$ of $B$ such that $T_{e}: B \rightarrow B(H)$ is compact. Notice now that if $\left(x_{n}\right)$ is any sequence of elements in $B$ for which $e x_{n}$ converges uniformly in $B(H)$, then

$$
\lim e x_{n}=\lim e\left(x_{n} e+e x_{n}-e x_{n} e\right)=e \lim \left(x_{n} \circ e-U_{e}\left(x_{n}\right)\right)
$$

which again lies in $e B$. It follows that $e B$ is of finite dimension, since it is uniformly closed and has compact identity operator. The weak operator closure $M$, of $B$ is a Type I $J W$-factor, by the results of [4], for example. Since $e M$ must be of finite dimension, so 
also must be the space generated by $\left\{U_{s}(e) ; s\right.$, a symmetry in $\left.M\right\}$. It follows, therefore, from [2, Theorem 6.10, Proposition 8.3], that $M$, and hence $B$, is of finite dimension. This completes the proof.

In conclusion, the following corollary (of which (ii), it should be said, was announced without proof in [4] and is a generalisation of a result of Kaplansky [13], on $C^{*}$ algebras) may be deduced by inspection of the above proof.

Corollary 6. Let $A$ be a JB-algebra. Then

(i) $C_{i}(J)=C_{i}(A) \cap J, i=1,2,3,4$, for every hereditary $J B$-subalgebra $J$ of $A$,

(ii) $L_{x}: A \rightarrow A$ is compact for every $x$ in $A$ if and only if $A$ is the $J B-(\infty)$ sum of finite dimensional $J B$-algebras.

Much of this work appears in the author's Ph.D. thesis, written at the University of Reading under the supervision of Professor J. D. M. Wright, and the author would like to acknowledge this fact.

\section{REFRENCES}

1. J. A. Alexander, Compact Banach algebras, Proc. London Math. Soc. (3) 18 (1968), 1-18.

2. E. M. Alfsen, F. W. Shultz and E. Stormer, A Gelfand Neumark theorem for Jordan algebras, Advances in Math. 28 (1978), 11-56.

3. H. BeHNCKE, Hermitian Jordan Banach algebras, J. London Math. Soc. (2) 20 (1979), 306-312.

4. L. J. BunCE, Theory and structure of dual $J B$-algebras, Math. Zeit. (to appear).

5. J. Dixmier, $C^{*}$-Algebras (North-Holland, Amsterdam, 1977).

6. N. Dunford and J. T. Schwarz, Linear Operators, Part I (Interscience, 1958).

7. C. M. Edwards, Ideal theory in JB-algebras, J. London Math. Soc. (2) 16 (1977), 507-513.

8. C. M. Edwards, On the facial structure of a JB-algebra, J. London Math. Soc. (2) 19 (1979), 335-344.

9. C. M. Edwards, On the centres of hereditary $J B W$-subalgebras of a $J B W$-algebra, Math. Proc. Cambs. Philos. Soc. 85 (1979), 317-325.

10. E. Efrros and E. Stormer, Jordan algebras of self adjoint operators, Trans. Amer. Math. Soc. 127 (1967), 312-315.

11. H. HANCHE-Olsen, A note on the bidual of a JB-algebra, Math. Zeit. 175 (1980), 29-31.

12. N. JACOBSON, Structure and Representations of Jordan Algebras (Amer. Math. Soc. Colloq. Publications 39, Providence R.I. 1968).

13. I. KaPlansKy, Dual rings, Duke Math. J. 6 (1949), 399-418.

14. R. R. SMrth, On non-unital Jordan Banach algebras, Math. Proc. Cambs. Philos. Soc. 82 (1977), 375-380.

15. F. W. Shultz, On normed Jordan algebras which are Banach dual spaces, J. Functional Analysis 31 (1979), 360-376.

16. D. Topping, Jordan Algebras of Self Adjoint Operators (Mem. Amer. Math. Soc. 53, 1965). 
17. D. ToppING, An isomorphism invariant for spin factors, J. Math. Mech. 15 (1965), 10551063.

18. J. D. M. WRIGHT, Jordan $C^{*}$-algebras, Michigan Math. J. 24 (1977), 291-302.

19. M. A. Youngson, Hermitian operators on Banach Jordan algebras, Proc. Edinburgh Math. Soc. 27 (1979), 169-180.

Mathematics Department

UNIVERSITY OF READING 